\title{
The Hybrid SPECT/CT as an Additional Lymphatic Mapping Tool in Patients with Breast Cancer
}

\author{
Iris M. C. van der Ploeg • Renato A. Valdés Olmos • \\ Bin B. R. Kroon · Omgo E. Nieweg
}

Published online: 14 May 2008

(C) The Author(s) 2008

\begin{abstract}
Background Conventional lymphoscintigraphy does not always define the exact anatomic location of a sentinel node. The lymphatic drainage pattern may be unusual or may not be shown at all. The recently introduced hybrid SPECT/CT imaging could help overcome these difficulties. SPECT is a tomographic version of conventional lymphoscintigraphy and the images have better contrast and resolution. When fused with the anatomical details provided by CT into one image, a meaningful surgical "roadmap" can be created. So far, there is little literature on the use of hybrid SPECT/CT in lymphatic mapping in patients with breast cancer. The purpose of this review was to report on these publications, including our own experience, focusing on patient selection, SPECT/CT settings, anatomic localization, and the detection of additional sentinel nodes.

Methods The majority of investigators did not formulate indications for additional SPECT/CT after conventional imaging but scanned all patients eligible for sentinel node biopsy. The SPECT/CT settings used in the studies of this review were mostly similar, but the methods used for conventional imaging were more variable.

Results All studies demonstrated an improved anatomical localization by performing additional SPECT/CT; sentinel nodes outside the axilla or nodes close to the injection site
\end{abstract}

I. M. C. van der Ploeg $(\bowtie)$ - B. B. R. Kroon · O. E. Nieweg Department of Surgery, The Netherlands Cancer InstituteAntoni van Leeuwenhoek Hospital, Plesmanlaan 121, 1066 CX Amsterdam, The Netherlands

e-mail: i.vd.ploeg@nki.nl

R. A. Valdés Olmos

Department of Nuclear Medicine, The Netherlands Cancer Institute-Antoni van Leeuwenhoek Hospital, 1066 CX Amsterdam, The Netherlands were especially easier to identify. Sentinel nodes were visualized in $89-100 \%$ by combined conventional imaging and SPECT/CT, with sentinel nodes depicted only by SPECT/CT in up to $14 \%$.

Conclusion It is concluded that SPECT/CT shows the exact anatomical location of sentinel nodes, detects sentinel nodes not depicted by conventional imaging, and therefore facilitates surgical exploration. The hybrid SPECT/CT has the potential to make image fusion a routine clinical tool that improves lymphatic mapping in patients with breast cancer.

\section{Introduction}

Conventional lymphoscintigraphy does not always define the exact anatomic location of a sentinel node [1-4]. Lymphatic drainage may follow an unusual pattern or may not be shown at all on conventional images. The intraoperative use of blue dye and a hand-held gamma-ray detection probe still enables identification of the sentinel node in many of such patients but not in all of them [5]. A noninvasive technique that allows for an improved preoperative sentinel node localization and detection rate would be helpful.

The combination of a SPECT (single-photon emission computed tomography) camera and a CT integrates physiologic and anatomic information [6]. SPECT is a tomographic version of conventional scintigraphy, in this case lymphoscintigraphy, but with a better contrast and resolution. In combination with the anatomical details provided by $\mathrm{CT}$, a meaningful roadmap for the surgeon can be obtained.

Initially, attempts were made to combine separately performed SPECT and CT images after acquisition of these 
two independent imaging modalities [7, 8]. Problems in patient preparation and mathematical modeling made proper alignment of the two techniques too time-consuming for a routine procedure.

The recently introduced hybrid SPECT/CT unites both imaging techniques in one device, which is beneficial in several ways [9-11]. Special computer software and external fusion landmarks are redundant. The position of the patient does not need to be changed, which avoids possible errors in image alignment caused by movement of internal organs. Scatter and attenuation induced by the generation or fusion of the SPECT and CT images also can be corrected.

So far, the available literature on the use of a hybrid SPECT/CT in lymphatic mapping in patients with cancer is limited. The purpose of this literature study was to review the publications that have explored the additional value of SPECT/CT in lymphatic mapping in patients with breast cancer, including our own experience, focusing on patient selection, SPECT/CT settings, anatomic localization, and the detection of additional sentinel nodes.

\section{Methods}

The databases PubMed, Embase, and the Cochrane Library were searched for studies concerning patients with breast cancer who underwent SPECT/CT for lymphatic mapping. The following medical subject headings (MeSH) terms were used to find relevant articles: "breast neoplasms"; "sentinel lymph node biopsy"; and "tomography, emission-computed, single-photon." All data concerning patient selection, SPECT/CT settings, anatomic localization, and the detection of additional sentinel nodes were analyzed.

\section{Results}

Four studies were published between March 2006 and November 2007, which described a total of 449 patients, of which 351 are described in this article (Tables 1 and 2) [12-15]. Lerman et al. has twice published on the use of SPECT/CT in lymphatic mapping in patients with breast cancer [13, 14]. The first publication is included in this report and only the overweight patient group from the second publication, because the remaining patients had already been described in the first paper. SPECT/CT was used for lymphatic mapping in consecutive patients with breast cancer in three institutes [12-14]. Lerman et al. [14] described the additional value in the above-mentioned subgroup of overweight patients. Van der Ploeg et al. [15] performed SPECT/CT only as additional technique in difficult cases, such as conventional images with lymphatic drainage to nodes outside the axilla, sentinel nodes close to the injection site, or images without a sentinel node. SPECT/CT was performed without reinjection of the radiopharmaceutical in this study.

The protocols for conventional imaging differed from each other (Table 1). Dosages of $74 \mathrm{MBq}$ to $120 \mathrm{MBq}$ ${ }^{99} \mathrm{~m}$ Tc-labeled tracers were administered into or around the tumor and early imaging was performed. Additional late images were obtained in three of the four studies. All papers described the use of a hybrid SPECT/CT with a low-dose CT. A 2-day protocol was used with lymphoscintigraphy on the first day and the operation on the second. The SPECT settings and acquisition times mentioned in the papers were similar (Table 2). All groups of investigators performed SPECT/CT after conventional imaging and corrected for attenuation and scatter.

All four papers mention improved anatomical localization by performing additional SPECT/CT after conventional imaging [12-15]. Husarik et al. [12] describe that SPECT/CT provided more accurate anatomical information in $82 \%$ of patients and was conclusive in exact anatomical information in 70\%. SPECT/CT also discovered additional sites of lymphatic drainage not depicted by conventional scans. Overall, sentinel nodes were visualized on conventional images in 72-94\% of the patients and in 89-100\% when SPECT/CT was added. The improvement was most pronounced in problematic cases. In $4-14 \%$ of

Table 1 Patients and methods of conventional imaging

\begin{tabular}{|c|c|c|c|c|c|c|}
\hline Study & Date & $\begin{array}{l}\text { No. of } \\
\text { patients }\end{array}$ & Indication & Dosage and radiotracer & $\begin{array}{l}\text { Injection } \\
\text { site }\end{array}$ & Conventional imaging \\
\hline Lerman et al. [13] & March 2006 & 157 & T0-2N0M0 & $74 \mathrm{MBq}{ }^{99 m}$ Tc-rhenium colloid & PT or IT & $60 \mathrm{~min}$ up to $24 \mathrm{hr}$ p.i. \\
\hline Husarik et al. [12] & January 2007 & 41 & T0-2N0M0 & $80 \mathrm{MBq}{ }^{99 m} \mathrm{Tc}$-nanocolloid & PT and ID & $30 \min$ p.i. \\
\hline Lerman et al. [14] & Febraury 2007 & 122 & Overweight patients & $74 \mathrm{MBq}{ }^{99 m}$ Tc-rhenium colloid & PT or IT & $60 \mathrm{~min}$ up to $24 \mathrm{hr}$ p.i. \\
\hline $\begin{array}{l}\text { Van der Ploeg } \\
\text { et al. [15] }\end{array}$ & November 2007 & 31 & $\begin{array}{l}\text { Unusual conventional } \\
\text { images }^{\mathrm{a}}\end{array}$ & $120 \mathrm{MBq}{ }^{99 \mathrm{~m}} \mathrm{Tc}-$ nanocolloid & IT & $15 \mathrm{~min}, 2,4 \mathrm{hr}$ p.i. \\
\hline
\end{tabular}

${ }^{a}$ Conventional images that were difficult to interpret, such as sentinel nodes outside the axilla or nonvisualization

$P T$ peritumoral, IT intratumoral, ID intradermal, p.i. postinjection 
Table 2 Methods and results of SPECT/CT imaging

\begin{tabular}{|c|c|c|c|c|c|}
\hline Study & $\begin{array}{l}\text { SPECT/CT } \\
\text { after CI }\end{array}$ & SPECT & $\begin{array}{l}\text { Acquisition } \\
\text { time (min) }\end{array}$ & $\begin{array}{l}\text { SN visualization } \\
\text { CI/SPECT/CT (\%) }\end{array}$ & $\begin{array}{l}\text { SNs only } \\
\text { on SPECT (\%) }\end{array}$ \\
\hline Lerman et al. [13] & Yes $^{\mathrm{a}}$ & $\begin{array}{l}\text { Matrix } 128 \times 128,3^{\circ} \text { - angle steps, } \\
20 / 25 \text { s time frame }\end{array}$ & $25-28$ & $84 / 91$ & 13 \\
\hline Husarik et al. [12] & Yes, 40 min p.i. & $\begin{array}{l}\text { Matrix } 128 \times 128,3^{\circ} \text { - angle steps, } \\
20 \mathrm{~s} \text { time frame }\end{array}$ & 27 & Not mentioned & 14 \\
\hline Lerman et al. [14] & Yes $^{\mathrm{a}}$ & $\begin{array}{l}\text { Matrix } 512 \times 512,3^{\circ} \text { - angle steps, } \\
20 / 25 \text { s time frame }\end{array}$ & $25-28$ & $72 / 89$ & Not mentioned \\
\hline $\begin{array}{l}\text { Van der Ploeg } \\
\text { et al. [15] }\end{array}$ & Yes, 4 hr p.i. & $\begin{array}{l}\text { Matrix } 128 \times 128,6^{\circ} \text { - angle steps, } \\
25 \text { s time frame }\end{array}$ & 15 & $94 / 100$ & 4 \\
\hline
\end{tabular}

${ }^{\text {a }}$ SPECT/CT was presumably performed 60 minutes after radiopharmaceutical injection and sometimes before conventional imaging $C I$ conventional imaging, $S N$ sentinel node, p.i. postinjection

the patients, sentinel nodes were depicted only by SPECT/ CT (Fig. 1). In the study by Van der Ploeg et al. [15], 17\% of sentinel nodes visualized only by SPECT/CT were tumor-positive, which led to upstaging in these patients and changed their management. The additional sentinel nodes were depicted close to the radiopharmaceutical injection site, in the internal mammary chain, in the breast or between the pectoral muscles. In patients with internal mammary sentinel nodes, SPECT/CT can show the exact intercostal space or a location behind a rib (Fig. 2) [15]. SPECT/CT was able to distinguish two sentinel nodes from one another when depicted as one node on conventional images. Two papers mention that $4-17 \%$ of the hot spots detected on conventional scans were mistaken for nodes
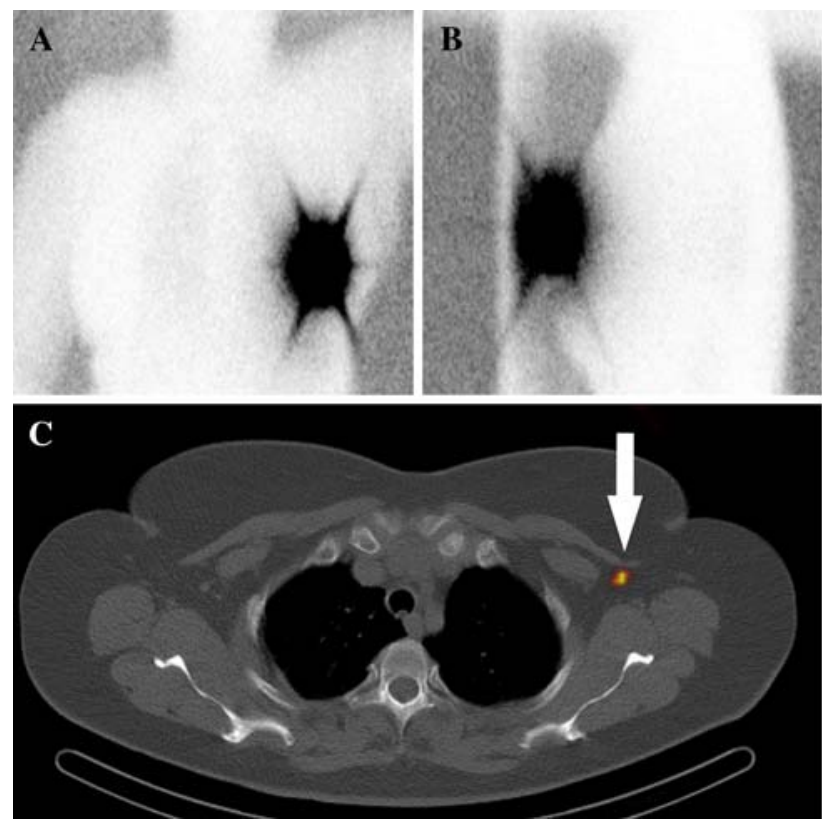

Fig. 1 Woman with a left breast cancer. The conventional lymphoscintigraphic anterior (A) and lateral (B) images 4 hours after radiopharmaceutical injection show no lymphatic drainage. The transaxial SPECT/CT (C) depicts an axillary sentinel node (arrow) but were accurately classified by SPECT/CT as non-nodal sites of uptake $[12,13]$. These false-positive sites of uptake were mostly the result of contamination of the skin with the radiopharmaceutical or the injection site was mistaken for a sentinel node. Lerman et al. [13] reported one patient $(0.6 \%)$ with a tumor-positive sentinel node that was visualized by conventional imaging and not depicted by SPECT/CT.

\section{Discussion}

Sentinel node biopsy in patients with clinically node-negative breast cancer is a valuable procedure for nodal staging. It allows the detection of clinically occult metastases by a meticulous histopathological examination, which may indicate the need for further treatment [16-18]. Accurate visualization of the sentinel nodes is required for the best results. Based on the concept of stepwise spread of metastases through the lymphatic system, all visualized nodes on a direct drainage pathway from the primary tumor site are sentinel nodes and should be identified and examined [19-21]. SPECT/CT was introduced in lymphatic mapping with the goal to show more sentinel nodes and to show them more clearly than is possible with conventional lymphoscintigraphy to improve nodal staging.

The SPECT/CT settings used in the studies in this review were comparable, but the methods used for conventional imaging were not always similar [12-15]. Especially the timing and acquisition duration of conventional scans and the timing of SPECT/CT imaging differed among the studies. The majority of investigators did not formulate indications for the use of SPECT/CT. SPECT/CT correlates radiotracer accumulation with a morphologic structure. Importantly, all four groups of investigators corrected for attenuation and scatter for the SPECT and CT images.

All studies mentioned better anatomical localization by performing additional SPECT/CT after conventional 

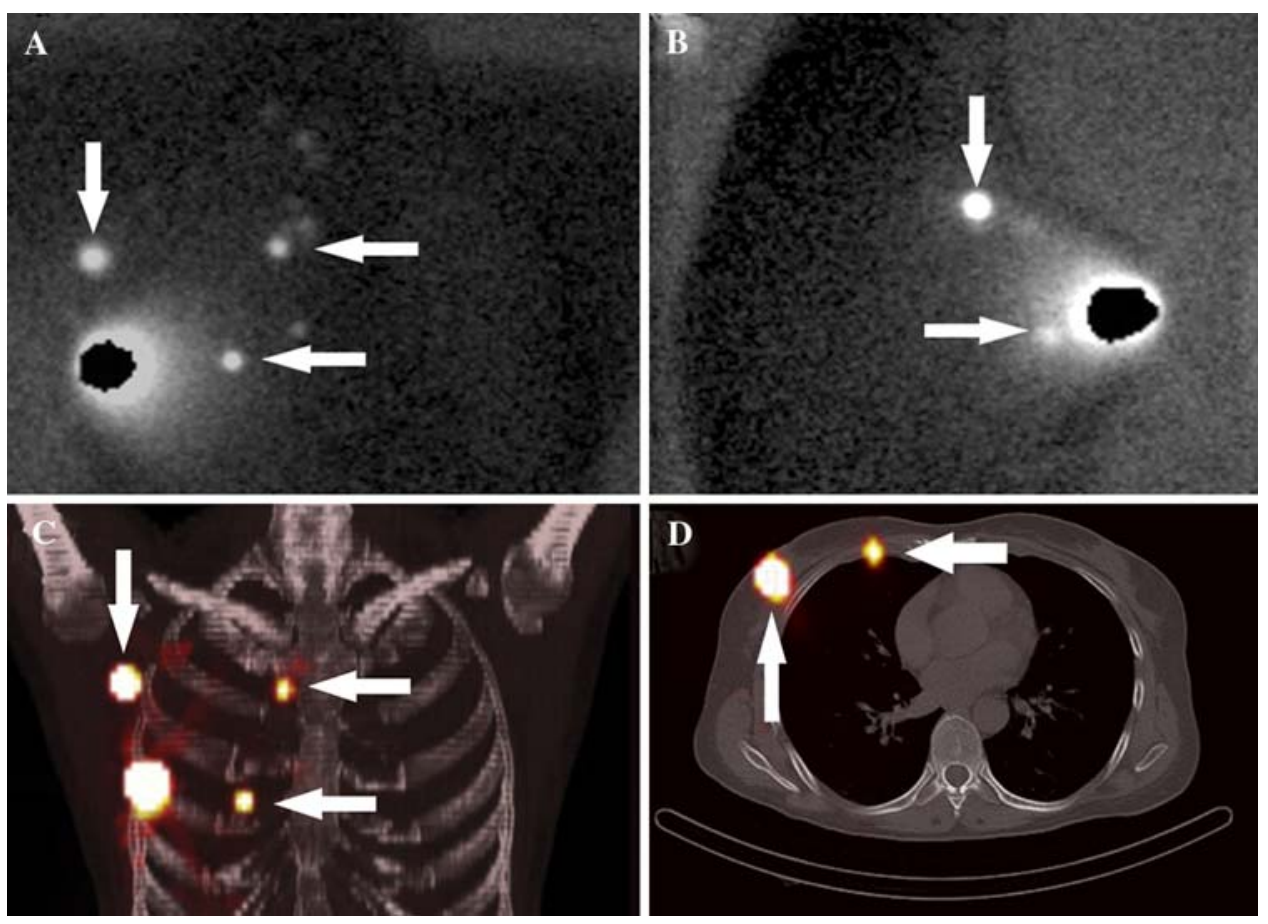

Fig. 2 Woman with a right breast cancer. The conventional lymphoscintigraphic anterior (A) and lateral (B) images show sentinel nodes in the axilla (descending arrow), in the internal mammary chain (upper horizontal arrow), and a sentinel node that is located in the breast or the internal mammary chain (lower horizontal arrow). The three-dimensional fused SPECT/CT maximum intensity projection (C) depicts the axillary sentinel node (descending arrow), the internal

imaging, which improved the surgical approach. Especially sentinel nodes outside the axilla or nodes close to the injection site were better located by performing additional SPECT/CT. Sentinel nodes were visualized on conventional images in $72-94 \%$ of the patients and these numbers improved to $89-100 \%$ by adding SPECT/CT. Sentinel nodes missed by conventional imaging were depicted by SPECT/CT in up to $14 \%$ of the cases. SPECT/CT showed that some sites of uptake that had been suggested to be sentinel nodes by conventional imaging were in reality something else, for example, sites of skin contamination with the radiopharmaceutical.

Failure of lymphoscintigraphic imaging in the reviewed papers might be caused by the technique that was used. For example, the dosage, volume, or particle size of the radiotracer might not have been the perfect combination to visualize all lymphatic drainage sites. The time of conventional imaging after injection of the radiotracer might not be ideal either. Some nuclear medicine physicians stopped data acquisition after early imaging, which prevents the visualization of sentinel nodes in case of slow lymph flow. SPECT/CT has been shown to improve the visualization rate because of its better contrast and resolution. mammary chain node in the second intercostal space (upper horizontal arrow), and shows that the other sentinel node is presumably located in the internal mammary chain (lower horizontal arrow). The transaxial fused SPECT/CT image (D) visualizes the axillary sentinel node (ascending arrow) and confirms that the lower sentinel node is an internal mammary chain node located in the fourth intercostal space (horizontal arrow)

The introduction of the hybrid SPECT/CT in daily practice is associated with extra costs and takes more time because it is additional to conventional lymphoscintigraphy. We think that the advantages prevail if SPECT/CT is used in problematic cases only.

Although the overall extent of the improvement is hard to establish because of differences in methods and patient selection, we conclude that the hybrid SPECT/CT takes preoperative lymphoscintigraphy to a higher level and has the potential to make image-fusion a routine clinical tool that will result in better staging of patients with breast cancer.

Open Access This article is distributed under the terms of the Creative Commons Attribution Noncommercial License which permits any noncommercial use, distribution, and reproduction in any medium, provided the original author(s) and source are credited.

\section{References}

1. Goyal A, Newcombe RG, Mansel RE et al (2005) Role of routine preoperative lymphoscintigraphy in sentinel node biopsy for breast cancer. Eur J Cancer 41:238-243

2. Krausz Y, Ikeda DM, Jadvar $\mathrm{H}$ et al (2001) Nonvisualization of sentinel lymph node in patients with breast cancer. Nucl Med Commun 22:25-32 
3. Valdes-Olmos RA, Jansen L, Hoefnagel CA et al (2000) Evaluation of mammary lymphoscintigraphy by a single intratumoral injection for sentinel node identification. J Nucl Med 41:15001506

4. Valdes Olmos RA, Tanis PJ, Hoefnagel CA et al (2001) Improved sentinel node visualization in breast cancer by optimizing the colloid particle concentration and tracer dosage. Nucl Med Commun 22:579-586

5. Tanis PJ, van Sandick JW, Nieweg OE et al (2002) The hidden sentinel node in breast cancer. Eur J Nucl Med Mol Imaging 29:305-311

6. Keidar Z, Israel O, Krausz Y (2003) SPECT/CT in tumor imaging: technical aspects and clinical applications. Semin Nucl Med 33:205-218

7. Kizu H, Takayama T, Fukuda M et al (2005) Fusion of SPECT and multidetector CT images for accurate localization of pelvic sentinel lymph nodes in prostate cancer patients. J Nucl Med Technol 33:78-82

8. Kretschmer L, Altenvoerde G, Meller J et al (2003) Dynamic lymphoscintigraphy and image fusion of SPECT and pelvic CTscans allow mapping of aberrant pelvic sentinel lymph nodes in malignant melanoma. Eur J Cancer 39:175-183

9. Even-Sapir E, Lerman H, Lievshitz G et al (2003) Lymphoscintigraphy for sentinel node mapping using a hybrid SPECT/CT system. J Nucl Med 44:1413-1420

10. Khafif A, Schneebaum S, Fliss DM et al (2006) Lymphoscintigraphy for sentinel node mapping using a hybrid single photon emission CT (SPECT)/CT system in oral cavity squamous cell carcinoma. Head Neck 28:874-879

11. Sherif A, Garske U, de la Torre M et al (2006) Hybrid SPECTCT: an additional technique for sentinel node detection of patients with invasive bladder cancer. Eur Urol 50:83-91
12. Husarik DB, Steinert HC (2007) Single-photon emission computed tomography/computed tomography for sentinel node mapping in breast cancer. Semin Nucl Med 37:29-33

13. Lerman H, Metser U, Lievshitz G et al (2006) Lymphoscintigraphic sentinel node identification in patients with breast cancer: the role of SPECT-CT. Eur J Nucl Med Mol Imaging 33:329-337

14. Lerman H, Lievshitz G, Zak O et al (2007) Improved sentinel node identification by SPECT/CT in overweight patients with breast cancer. J Nucl Med 48:201-206

15. Van der Ploeg IM, Valdes Olmos RA, Nieweg OE et al (2007) The additional value of SPECT/CT in lymphatic mapping in breast cancer and melanoma. J Nucl Med 48(11):1756-1760

16. Krag DN, Anderson SJ, Julian TB et al (2007) Technical outcomes of sentinel-lymph-node resection and conventional axillary-lymph-node dissection in patients with clinically nodenegative breast cancer: results from the NSABP B-32 randomised phase III trial. Lancet Oncol 8:881-888

17. Mansel RE, Fallowfield L, Kissin M et al (2006) Randomized multicenter trial of sentinel node biopsy versus standard axillary treatment in operable breast cancer: the ALMANAC Trial. J Natl Cancer Inst 98:599-609

18. Veronesi U, Paganelli G, Viale G et al (2003) A randomized comparison of sentinel-node biopsy with routine axillary dissection in breast cancer. N Engl J Med 349:546-553

19. Kapteijn BA, Nieweg OE, Petersen JL et al (1998) Identification and biopsy of the sentinel lymph node in breast cancer. Eur J Surg Oncol 24:427-430

20. Nieweg OE, Tanis PJ, Kroon BB (2001) The definition of a sentinel node. Ann Surg Oncol 8:538-541

21. Reintgen D, Cruse CW, Wells K et al (1994) The orderly progression of melanoma nodal metastases. Ann Surg 220:759-767 